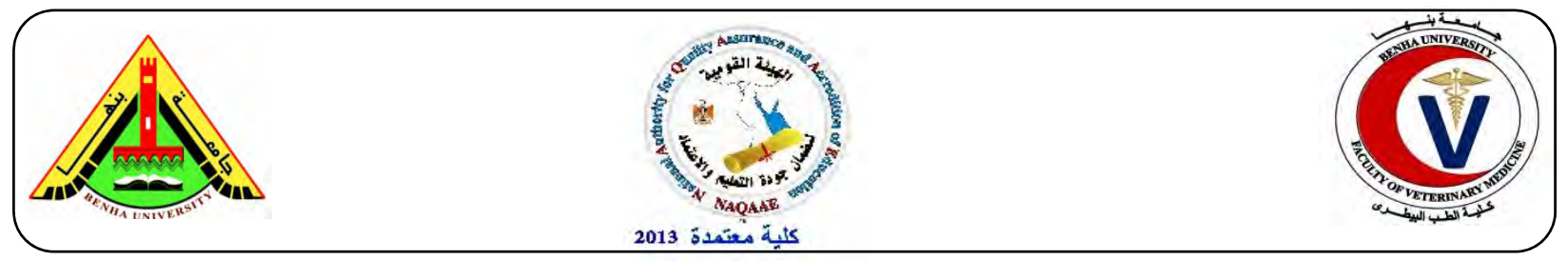

\title{
Genetic evaluation for growth traits in Japanese quail.
}

Eman A. Manaa ${ }^{1}$, Khairy M. El-Bayomi ${ }^{2}$, Gamal A. Sosa ${ }^{3}$

${ }^{1}$ Department of animal wealth, Faculty of Veterinary Medicine, Benha University. ${ }^{2}$ Department of animal wealth, Faculty of Veterinary Medicine, Zagazig University. ${ }^{3}$ Department of Theriogenoology, Faculty of Veterinary Medicine, Benha University.

\section{A B S T R A C T}

The main objective of this work was to evaluate the means and genetic parameters for growth traits including body weight, average daily gain (ADG) and relative growth rate (RGR) of random population of Japanese quail. The second generation was higher than the first generation at hatching, 1st, 2nd and 3rd week of age $(7.28,28.14,52.70$ and $88.48 \mathrm{~g}$; respectively). However, the base generation showed the highest values at 4th week, 5th week and 6th week of age $(173.25,189.53$ and $205.23 \mathrm{~g}$; respectively). The second generation had the highest values of ADG at hatching-1st, 1st -2nd, 2nd 3rd, 4th -5th and 5th -6th week intervals $(3.03,3.50,5.13,4.01$ and $2.72 \mathrm{~g}$; respectively). Nevertheless, the first generation recorded the highest values of ADG at 3rd-4th week interval $(5.22 \mathrm{~g})$. The second generation had the highest values of RGR at hatching-1 st, 1st -2nd, 2nd -3rd, 4th -5th, 5th -6th week intervals $(116.99,61.69,52.60,22.12$ and $12.78 \%$; respectively). However, the first generation recorded the highest values of RGR at 3rd -4th week intervals (36.80\%).

Keywords: Japanese quail, Body weight, Average daily gain, Relative growth rate, Heritability.

(http:// www.bvmj.bu.edu.eg)

(BVMJ-28(1): 8-16, 2015)

\section{INTRODUCTION}

Japanese quail is the smallest avian species raised for meat and egg production (Panda and Singh, 1990) and it has also assumed world-wide importance as a laboratory animal (Baumgartner, 1990). Establishment of breeding programs necessitates estimation of the genetic parameters for different productive and reproductive traits. Japanese quail is suggested as a pilot animal for genetic studies because of its shorter generation interval and the economy of production resulting from faster growth rate and its smaller body size (Devi et al., 2010). Genetic studies on Japanese quail in Egypt will enable breeders to design suitable improvement programs for this bird. Therefore, reliable estimates of genetic parameters (heritability and correlations) are necessary to predict the direct and indirect selection responses (Harvey and Bearden, 1962). The aim of this study was to evaluate the means and genetic parameters for growth traits including body weight, average daily gain (ADG) and relative growth rate (RGR) of random mating population of Japanese quail.

\section{MATERIAL AND METHODS}

\subsection{Management of the birds}

\subsubsection{Flock Managements of the birds}

Base generation was randomly allotted to 57 sire families and labeled each bird by colored wing band and sire families were housed in wire cages $(25 \times 25 \times 25 \mathrm{~cm})$ with sex ratio 1 male: 2 female. Sixteen hours lighting period was adjusted during the laying period. Chicks were floor brooded at $36{ }^{\circ} \mathrm{C}$ at the bird level. Temperature was decreased gradually by $3{ }^{\circ} \mathrm{C}$ weekly till reach $24^{\circ} \mathrm{C}$ at the fourth week. Lighting was provided 24 hours daily till $4^{\text {th }}$ week of age then reduced to 14 hours of light and 19 hours of darkness. 
Table (A): Each number of hatches and number of sire families of generation.

\begin{tabular}{ccc}
\hline Generation & $\begin{array}{c}\text { Number of } \\
\text { hatches }\end{array}$ & $\begin{array}{c}\text { Number of sire } \\
\text { families }\end{array}$ \\
\hline Base & - & 57 \\
First & 3 & 57 \\
Second & 3 & 28 \\
\hline
\end{tabular}

\subsubsection{Feeding management}

Birds were fed ad libtium on diet containing 21 laying and $29 \%$ growing crude protein and $2975.8 \mathrm{Kcal} \mathrm{ME} / \mathrm{kg}$ of feed.

\subsubsection{Egg Incubation and hatching}

Eggs were collected daily after complete sexual maturity. Eggs tagged according to their sire families then stored at $18{ }^{\circ} \mathrm{C}$ for a week. Pedigreed eggs were set in the setting trays according to their sire families in a forced draft incubator at $37.5^{\circ} \mathrm{C}$ and 60 $70 \%$ relative humidity $(\mathrm{RH})$. Eggs were turned automatically every three hours. At the 14th day of incubation eggs were transferred in pedigree baskets to the hatchers where the temperature was $37.5^{\circ} \mathrm{C}$ and $\mathrm{RH}$ was $70 \%$.

\subsection{Studied traits and Estimations for base,} first, second generation

\subsubsection{Body weight}

At hatch, $1^{\text {st }}, 2^{\text {nd }}, 3^{\text {rd }}, 4^{\text {th }}, 5^{\text {th }}$ and $6^{\text {th }}$ week.

\subsubsection{Average daily gain}

It is the weight gain related to the number of days calculated.

\subsubsection{Relative growth rate}

Relative Growth Rate $($ RGR $)=\frac{W_{2}-W_{1}}{1 / 2\left(W_{2}+W_{1}\right)} \times 100$

Where: - W1: body weight at the beginning of period and W2: body weight at the end of period (Broody, 1945).

\subsubsection{Genetic parameters}

Heritability estimate
It was calculated from sire component of variance per generation according to the following formula Becker (1985):

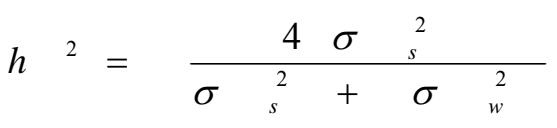

Where:

$$
\begin{aligned}
& \sigma_{s}^{2}=\text { Sire variance components. } \\
& \sigma_{w}^{2}=\text { within sire residual variance components. }
\end{aligned}
$$

\section{Correlations}

\section{a. Phenotypic correlation: " $\mathbf{r}_{\mathbf{P}}$ "}

Calculated according to the following formula Becker (1985):

$$
r_{P}=\frac{\operatorname{cov} s+\operatorname{cov} w}{\sqrt{\left[( \sigma _ { s } ^ { 2 } ( x ) + \sigma _ { w } ^ { 2 } ( x ) ] \left[\left(\sigma_{s}^{2}(y)+\sigma_{w}^{2}(y)\right]\right.\right.}}
$$

Where:

$\mathrm{Cov}_{\mathrm{s}}=$ sire covariance components.

$\mathrm{Cov}_{\mathrm{w}}=$ within sire covariance components.

$\sigma_{s(x)}^{2}=$ sire variance components for trait (x).

$\sigma_{s(y)}^{2}=$ Sire variance components for trait (y).

$\sigma_{w(x)}^{2}=$ within sire variance components for trait (x).

$\sigma_{w(y)}^{2}=$ within sire variance components for trait (y).

\section{b. Genetic correlation: "rG"}

Calculated according to the following formula Becker (1985):

$$
r_{G}=\frac{C O V s}{\sqrt{\sigma_{S}^{2}(x) x \sigma_{S}^{2}(y)}}
$$

Where:

$\mathrm{COV}_{\mathrm{s}}=$ Sire covariance components.

$$
\begin{aligned}
& \sigma_{s(x)}^{2}=\text { Sire variance components for trait. } \\
& \sigma_{s(y)}^{2}=\text { Sire variance components for trait }(\mathrm{y})
\end{aligned}
$$

\subsection{Data handling and statistical analysis}

Statistical analysis was carried out using SAS statistical analysis system package software (SAS, 2002) according to the following models. 
- Means for all traits under investigation

Where:

$$
X_{i j}=\mu+G_{i}++e_{i j}
$$

$\mathrm{Xij}=$ the Xth observation of the ith generation.

$\mu=$ overall mean.

$\mathrm{gi}=$ effect of ith generation $(\mathrm{i}=0,1,2)$.

eij $=$ random error.

\section{- Heritability and correlations}

$$
X_{i j}=\mu+S_{i}+e_{i j}
$$

Where:

$\mathrm{Xij}=$ the trait.

$\mu=$ population mean.

$\mathrm{Si}=$ effect of $\mathrm{i}^{\text {th }}$ sire.

eij $=$ uncontrolled environmental and genetic deviations.

Variance and covariance components for heritability and correlation were determined by SAS program, using Proc Nested and proc Var Comp (SAS, 2002).

\section{RESULTS}

On body weights at different ages studied, the second generation was higher than the first generation at hatching, $1^{\text {st }}$ week, $2^{\text {nd }}$ week and $3^{\text {rd }}$ week of age. But, the base generation showed the highest values at $4^{\text {th }}$ week, $5^{\text {th }}$ week and $6^{\text {th }}$ week of age (Table 1).

ADG at different ages' intervals studied, the second generation had the highest values of ADG at hatching- $1^{\text {st }}, 1^{\text {st }}-2^{\text {nd }}, 2^{\text {nd }}-3^{\text {rd }}, 4^{\text {th }}-$ $5^{\text {th }}$ and $5^{\text {th }}-6^{\text {th }}$ week intervals. But, the first generation recorded the highest values of ADG at $3^{\text {rd }}$ week $-4^{\text {th }}$ week interval (Table 2). On RGR at different ages' intervals studied, the second generation had the highest values of RGR at hatching- $1^{\text {st }}, 1^{\text {st }}$ $2^{\text {nd }}, 2^{\text {nd }}-3^{\text {rd }}, 4^{\text {th }}-5^{\text {th }}, 5^{\text {th }}-6^{\text {th }}$ week intervals. But, the first generation recorded the highest values of RGR at $3^{\text {rd }}-4^{\text {th }}$ week intervals (Table 3).

Tables (4 and 5) showed heritability estimates for body weights at different ages in the first and the second generation. All heritability estimates were high values except values for $5^{\text {th }}$ and $6^{\text {th }}$ in the first generation were low and also values for hatching and $4^{\text {th }}$ week in the first generation were moderate.

Phenotypic and genetic correlations for body weights in the first and the second generation showed positive high estimates except for genetic correlation between hatching and $6^{\text {th }}$ weights was moderate and also negative genetic correlation between $5^{\text {th }}$ and $6^{\text {th }}$ week body weights Tables (4) and (5).

Tables (6-7) showed heritability estimates for ADG at different age intervals in the first and the second generation. All heritability estimates were high values except values for 1 st- $2^{\text {nd }}$ and $2^{\text {nd }}-3^{\text {rd }}$ weeks intervals in the first generation were medium.

Phenotypic correlations in the first generation for ADG were positive values except between $5^{\text {th }}-6^{\text {th }}$ week interval and other ADG intervals and also negative values between $4^{\text {th }}-5^{\text {th }}$ week and hatching$1^{\text {st }}, 1^{\text {st }}-2^{\text {nd }}$ and $3^{\text {rd }}-4^{\text {th }}$ week's intervals. But, Genetic correlations in the first generation for ADG were negative values except between hatching- $1^{\text {st }}$ week and $1^{\text {st }}-2^{\text {nd }}$ week, hatching- $1^{\text {st }}$ week and $2^{\text {nd }}-3^{\text {rd }}$ week, $1^{\text {st }}-2^{\text {nd }}$ week and $2^{\text {nd }}-3^{\text {rd }}$ week, $3^{\text {rd }}-4^{\text {th }}$ week and $5^{\text {th }}$ $6^{\text {th }}$ week and $4^{\text {th }}-5^{\text {th }}$ and $5^{\text {th }}-6^{\text {th }}$ week (Table $6)$.

Phenotypic correlations in the second generation for ADG were positive values except between $5^{\text {th }}-6^{\text {th }}$ week interval and other ADG intervals and also negative values between $4^{\text {th }}-5^{\text {th }}$ week and other ADG intervals. Genetic correlations in the second generation between hatching- $1^{\text {st }}$ week ADG and other ADG intervals were positive except with $4^{\text {th }}-5^{\text {th }}$ week ADG was negative. Genetic correlations between $1^{\text {st }}$ $2^{\text {nd }}$ week ADG and other ADG intervals showed positive values except $4^{\text {th }}-5^{\text {th }}$ and $5^{\text {th }}-6^{\text {th }}$ week were negative. Genetic correlation for $2^{\text {nd }}-3^{\text {rd }}$ week and $3^{\text {rd }}-4^{\text {th }}$ week with other ADG intervals were negative but positive between $4^{\text {th }}-5^{\text {th }}$ week with $5^{\text {th }}-6^{\text {th }}$ week (Table 7).

All heritability estimates for RGR at different age intervals in the first and the 
second generation showed high values except values for hatching- $1^{\text {st }}$ and $2^{\text {nd }}-3^{\text {rd }}$ weeks intervals in the first generation showed low values and also were moderate values at $1^{\text {st }}-2^{\text {nd }}$ weeks intervals in the first generation and 2 nd- $3^{\text {rd }}$ weeks intervals in the second generation (Tables8-9).

Phenotypic correlations in the first generation for RGR showed negative values for hatching- $1^{\text {st }}$ week with other RGR week intervals but recorded positive values for $2^{\text {nd }}$ $-3^{\text {rd }}$ week with other RGR week intervals and also positive between $4^{\text {th }}-5^{\text {th }}$ week and $5^{\text {th }}-6^{\text {th }}$ week. Phenotypic correlations for $1^{\text {st }}$ $-2^{\text {nd }}$ week with other RGR week intervals were positive except with $5^{\text {th }}-6^{\text {th }}$ week. On the other hand Phenotypic correlation for $3^{\text {rd }}-4^{\text {th }}$ week was negative with $4^{\text {th }}-5^{\text {th }}$ week but positive with $5^{\text {th }}-6^{\text {th }}$ week (Table 8 ).

Genetic correlations in the first generation for RGR were positive values except between hatching- $1^{\text {st }}$ week and $1^{\text {st }}-2^{\text {nd }}$ week, hatching- $1^{\text {st }}$ week and $3^{\text {rd }}-4^{\text {th }}$ week, $1^{\text {st }}-2^{\text {nd }}$ week and $4^{\text {th }}-5^{\text {th }}$ week, $2^{\text {nd }}-3^{\text {rd }}$ week and $5^{\text {th }}-6^{\text {th }}$ week and $3^{\text {rd }}-4^{\text {th }}$ and $4^{\text {th }}-5^{\text {th }}$ week (Table 8).

Table (9) showed negative phenotypic correlations of RGR in the second generation for hatching- $1^{\text {st }}$ week with $2^{\text {nd }}-$ $3^{\text {rd }}$ week, hatching- $1^{\text {st }}$ week with $3^{\text {rd }}-4^{\text {th }}$ week, hatching- $1^{\text {st }}$ week with $4^{\text {th }}-5^{\text {th }}$ week, $1^{\text {st }}-2^{\text {nd }}$ week with $3^{\text {rd }}-4^{\text {th }}$ week and $3^{\text {rd }}-4^{\text {th }}$ week with $4^{\text {th }}-5^{\text {th }}$ week but other phenotypic correlation were positive. Meanwhile, negative genetic correlations of RGR in the second generation recorded for hatching- $1^{\text {st }}$ week with $2^{\text {nd }}-3^{\text {rd }}$ week, hatching $-1^{\text {st }}$ week with $4^{\text {th }}-5^{\text {th }}$ week, $1^{\text {st }}-2^{\text {nd }}$ week with $3^{\text {rd }}-4^{\text {th }}$ week, $1^{\text {st }}-2^{\text {nd }}$ week with $5^{\text {th }}-6^{\text {th }}$ week, $3^{\text {rd }}-4^{\text {th }}$ week with $4^{\text {th }}-5^{\text {th }}$ week and $3^{\text {rd }}-4^{\text {th }}$ week with $5^{\text {th }}-6^{\text {th }}$ week but other genetic correlation were positive.

Table (1): Least square means \pm standard errors ( $L S M \pm S E$ ) for generation effect on body weights of Japanese quails for two successive generations of random mating population.

\begin{tabular}{|c|c|c|c|c|c|c|c|}
\hline \multirow{2}{*}{$\begin{array}{c}\text { Trait } \\
\text { Generation }\end{array}$} & \multicolumn{7}{|c|}{ Body weight (g) (Mean \pm SE) /Age } \\
\hline & Hatching & $1^{\text {st }}$ week & $2^{\text {nd }}$ week & $3^{\text {rd }}$ week & $4^{\text {th }}$ week & $5^{\text {th }}$ week & $6^{\text {th }}$ week \\
\hline \multirow{2}{*}{ Base } & \multirow{2}{*}{-} & \multirow{2}{*}{-} & \multirow[b]{2}{*}{-} & \multirow{2}{*}{-} & $173.25^{\mathrm{a}} \pm$ & $189.53^{\mathrm{a}} \pm 1$ & $205.23^{a} \pm 1$ \\
\hline & & & & & 1.05 & 20 & .54 \\
\hline \multirow{2}{*}{ First } & \multirow{2}{*}{$7.00^{\mathrm{a}} \pm 0.14$} & $27.71^{\mathrm{a}}$ & $51.64^{\mathrm{a}}$ & $86.58^{\mathrm{a}}$ & $123.08^{\mathrm{b}} \pm$ & $141.24^{\mathrm{c}} \pm 2$ & $149.78^{\mathrm{c}} \pm 2$ \\
\hline & & \pm 0.73 & \pm 1.22 & \pm 1.83 & 2.19 & 28 & .78 \\
\hline \multirow{2}{*}{ Second } & \multirow{2}{*}{$7.28^{\mathrm{a}} \pm 0.19$} & $28.14^{\mathrm{a}}$ & $52.70^{\mathrm{a}}$ & $88.48^{\mathrm{a}}$ & $124.89^{\mathrm{b}} \pm$ & $148.99^{\mathrm{b}} \pm 3$ & $168.09^{\mathrm{b}} \pm 3$ \\
\hline & & \pm 0.99 & \pm 1.93 & \pm 2.78 & 3.35 & 33 & .12 \\
\hline
\end{tabular}

Means of different generations within the same column having different superscripts are significantly different $(\mathrm{p} \leq 0.05)$.

Table (2): Least square means \pm standard errors $(\mathrm{LSM} \pm \mathrm{SE})$ for generation effect on average daily gains (ADG) of Japanese quails for two successive generations of random mating population.

\begin{tabular}{|c|c|c|c|c|c|c|}
\hline Trait & & & G (g) (Mean & SE) /Age & & \\
\hline Generation & $\begin{array}{l}\text { Hatching- } 1^{\text {st }} \\
\text { week }\end{array}$ & $\begin{array}{l}1^{\text {st }} \text { week- } \\
2^{\text {nd }} \text { week }\end{array}$ & $\begin{array}{l}2^{\text {nd }} \text { week- } \\
3^{\text {rd }} \text { week }\end{array}$ & $\begin{array}{l}3^{\text {rd }} \text { week- } \\
4^{\text {th }} \text { week }\end{array}$ & $\begin{array}{l}4^{\text {th }} \text { week- } \\
5^{\text {th }} \text { week }\end{array}$ & $\begin{array}{l}5^{\text {th }} \text { week- } \\
6^{\text {th }} \text { week }\end{array}$ \\
\hline Base & - & - & - & - & $\begin{array}{c}2.95^{\mathrm{b}} \\
\pm 0.32\end{array}$ & $\begin{array}{l}2.24^{\mathrm{b}} \\
\pm 0.15\end{array}$ \\
\hline First & $2.91^{\mathrm{a}} \pm 0.07$ & $3.41^{\mathrm{a}} \pm 0.08$ & $4.98^{\mathrm{a}} \pm 0.11$ & $\begin{array}{c}5.22^{\mathrm{a}} \\
\pm 0.09\end{array}$ & $\begin{array}{c}3.43^{\mathrm{a}} \\
\pm 0.19\end{array}$ & $\begin{array}{l}2.22^{b} \\
\pm 0.11\end{array}$ \\
\hline Second & $3.03^{\mathrm{a}} \pm 0.09$ & $3.50^{\mathrm{a}} \pm 0.13$ & $5.13^{\mathrm{a}} \pm 0.18$ & $\begin{array}{l}5.21^{\mathrm{a}} \\
\pm 0.17\end{array}$ & $\begin{array}{l}4.01^{\mathrm{a}} \\
\pm 0.33\end{array}$ & $2.72^{\mathrm{a}} \pm 0.13$ \\
\hline
\end{tabular}

Means of different generations within the same column having different superscripts are significantly different $(\mathrm{p} \leq 0.05)$. 
Table (3): Least square means \pm standard errors ( $\mathrm{LSM} \pm \mathrm{SE}$ ) for generation effect on relative growth Rates (RGR) of Japanese quails for two successive generations of random mating population.

\begin{tabular}{|c|c|c|c|c|c|c|}
\hline Trait & & & $\mathrm{R}(\%) \quad(\mathrm{I}$ & $\pm \mathrm{SE}) / \mathrm{A}$ & & \\
\hline Generation & $\begin{array}{l}\text { Hatching- } \\
1^{\text {st }} \text { week }\end{array}$ & $\begin{array}{l}1^{\text {st }} \text { week- } \\
2^{\text {nd }} \text { week }\end{array}$ & $\begin{array}{l}2^{\text {nd }} \text { week- } \\
3^{\text {rd }} \text { week }\end{array}$ & $\begin{array}{l}3^{\text {rd }} \text { week- } \\
4^{\text {th }} \text { week }\end{array}$ & $\begin{array}{l}4^{\text {th }} \text { week- } \\
5^{\text {th }} \text { week }\end{array}$ & $\begin{array}{l}5^{\text {th }} \text { week- } \\
6^{\text {th }} \text { week }\end{array}$ \\
\hline Base & - & - & - & - & $\begin{array}{l}11.42^{\mathrm{b}} \\
\pm 1.26\end{array}$ & $\begin{array}{l}7.81^{\mathrm{b}} \\
\pm 0.52\end{array}$ \\
\hline First & $\begin{array}{c}113.72^{\mathrm{a}} \\
\pm 1.01\end{array}$ & $\begin{array}{l}61.31^{\mathrm{a}} \\
\pm 0.72\end{array}$ & $\begin{array}{l}51.98^{\mathrm{a}} \\
\pm 0.92\end{array}$ & $\begin{array}{c}36.80^{\mathrm{a}} \\
\pm 0.67\end{array}$ & $\begin{array}{l}19.39^{\mathrm{b}} \\
\pm 1.49\end{array}$ & $\begin{array}{l}11.78^{\mathrm{a}} \\
\pm 0.59\end{array}$ \\
\hline Second & $\begin{array}{c}116.99^{\mathrm{a}} \\
\pm 1.61\end{array}$ & $\begin{array}{l}61.69^{\mathrm{a}} \\
\pm 1.28\end{array}$ & $\begin{array}{l}52.60^{\mathrm{a}} \\
\pm 1.45\end{array}$ & $\begin{array}{l}35.39^{\mathrm{a}} \\
\pm 1.27\end{array}$ & $\begin{array}{l}22.12^{\mathrm{a}} \\
\pm 2.21\end{array}$ & $\begin{array}{l}12.78^{\mathrm{a}} \\
\pm 0.71\end{array}$ \\
\hline
\end{tabular}

Means of different generations within the same column having different superscripts are significantly different ( $\mathrm{p}$ $\leq 0.05)$.

Table (4): Heritability (on diagonal), phenotypic correlation (above diagonal) and genetic correlation (below diagonal) for body weights of Japanese quails in first generation of random mating population.

\begin{tabular}{cccccccc}
\hline Trait & & \multicolumn{5}{c}{ Body weight } \\
\hline Ages & Hatching & $1^{\text {st }}$ week & $2^{\text {nd }}$ week & $3^{\text {rd }}$ week & $4^{\text {th }}$ week & $5^{\text {th }}$ week & $6^{\text {th }}$ week \\
\hline Hatching & 0.29 & $0.87^{* *}$ & 0.80 & 0.73 & $0.65^{* *}$ & 0.63 & $0.61^{*}$ \\
$1^{\text {st }}$ week & $1.12^{* *}$ & 0.51 & $0.96^{* *}$ & $0.85^{* *}$ & $0.81^{* *}$ & $0.77^{* *}$ & $0.72^{* *}$ \\
$2^{\text {nd }}$ week & 1.25 & $1.00^{* *}$ & 0.40 & $0.90^{* *}$ & $0.85^{* *}$ & $0.82^{* *}$ & 0.75 \\
$3^{\text {rd }}$ week & 1.12 & $0.94^{* *}$ & $1.01^{* *}$ & 0.35 & $0.94^{* *}$ & $0.90^{* *}$ & $0.84^{* *}$ \\
$4^{\text {th }}$ week & $0.61^{* *}$ & $0.72^{* *}$ & $0.77^{* *}$ & $0.71^{* *}$ & 0.21 & $0.95^{* *}$ & $0.89^{* *}$ \\
$5^{\text {th }}$ week & 1.19 & $1.21^{* *}$ & $1.39^{* *}$ & $0.82^{* *}$ & $1.09^{* *}$ & 0.07 & $0.93^{* *}$ \\
$6^{\text {th }}$ week & $0.26^{*}$ & $0.86^{* *}$ & $1.00^{* *}$ & $0.23^{* *}$ & $0.53^{* *}$ & $-0.63^{* *}$ & 0.09 \\
\hline
\end{tabular}

* Significant at level (0.05), ** Highly significant at level (0.01).

Table (5): Heritability (on diagonal), phenotypic correlation (above diagonal) and genetic correlation (below diagonal) for body weights of Japanese quails in second generation of random mating population.

\begin{tabular}{lccccccc}
\hline Trait & \multicolumn{7}{c}{ Body weight } \\
\hline Ages & Hatching & $1^{\text {st }}$ week & $2^{\text {nd }}$ week & $3^{\text {rd }}$ week & $4^{\text {th }}$ week & $5^{\text {th }}$ week & $6^{\text {th }}$ week \\
\hline Hatching & 0.55 & 0.86 & 0.82 & 0.79 & 0.71 & 0.67 & 0.62 \\
$1^{\text {st }}$ week & 0.89 & 0.49 & $0.96^{* *}$ & $0.89^{* *}$ & $0.86^{* *}$ & $0.82^{* *}$ & $0.78^{* *}$ \\
$2^{\text {nd }}$ week & 0.95 & $1.01^{* *}$ & 0.44 & $0.93^{* *}$ & $0.89^{* *}$ & $0.85^{* *}$ & $0.80^{* *}$ \\
$3^{\text {rd }}$ week & 0.97 & $0.96^{* *}$ & $0.98^{* *}$ & 0.35 & $0.94^{* *}$ & $0.90^{* *}$ & $0.84^{* *}$ \\
$4^{\text {th }}$ week & 0.70 & $0.94^{* *}$ & $0.95^{* *}$ & $0.89^{* *}$ & 0.45 & $0.94^{* *}$ & $0.89^{* *}$ \\
$5^{\text {th }}$ week & 0.64 & $0.94^{* *}$ & $0.95^{* *}$ & $0.80^{* *}$ & $0.92^{* *}$ & 0.48 & $0.95^{* *}$ \\
$6^{\text {th }}$ week & 0.44 & $0.94^{* *}$ & $0.89^{* *}$ & $0.69^{* *}$ & $0.84^{* *}$ & $0.91^{* *}$ & 0.48 \\
\hline
\end{tabular}

* Significant at level (0.05), ** Highly significant at level (0.01). 
Genetic evaluation for growth traits in Japanese quail.

Table (6): Heritability (on diagonal), phenotypic correlation (above diagonal) and genetic correlation (below diagonal) for average daily gain (ADG) of Japanese quails in first generation of random mating population.

\begin{tabular}{|c|c|c|c|c|c|c|}
\hline Trait & \multicolumn{6}{|c|}{ ADG } \\
\hline Ages & $\begin{array}{l}\text { Hatching- } \\
1^{\text {st }} \text { week }\end{array}$ & $\begin{array}{l}1^{\text {st }} \text { week- } \\
2^{\text {nd }} \text { week }\end{array}$ & $\begin{array}{l}2^{\text {nd }} \text { week- } \\
3^{\text {rd }} \text { week }\end{array}$ & $\begin{array}{l}3^{\text {rd }} \text { week- } \\
4^{\text {th }} \text { week }\end{array}$ & $\begin{array}{l}4^{\text {th }} \text { week- } \\
5^{\text {th }} \text { week }\end{array}$ & $\begin{array}{l}5^{\text {th }} \text { week- } \\
6^{\text {th }} \text { week }\end{array}$ \\
\hline Hatching- $1^{\text {st }}$ week & 0.50 & 0.81 & $0.43^{*}$ & $0.10^{* *}$ & -0.06 & $-0.35^{* *}$ \\
\hline $1^{\text {st }}$ week- $2^{\text {nd }}$ week & 0.92 & 0.22 & $0.45^{* *}$ & $0.10^{* *}$ & $-0.03^{* *}$ & $-0.41^{*}$ \\
\hline $2^{\text {nd }}$ week- $3^{\text {rd }}$ week & $0.92^{*}$ & $1.67^{* *}$ & 0.10 & $0.02^{* *}$ & $0.05^{* *}$ & -0.26 \\
\hline $3^{\text {rd }}$ week- $4^{\text {th }}$ week & $-0.42^{* *}$ & $-0.59^{* *}$ & $-0.78^{* *}$ & 1.32 & $-0.15^{* *}$ & -0.10 \\
\hline $4^{\text {th }}$ week- $5^{\text {th }}$ week & -0.24 & $-0.34^{* *}$ & $-0.45^{* *}$ & $-0.55^{* *}$ & 1.32 & $-0.01^{*}$ \\
\hline $5^{\text {th }}$ week- $6^{\text {th }}$ week & $-0.16^{* *}$ & $-0.33^{*}$ & -1.17 & 0.17 & $0.23^{*}$ & 0.67 \\
\hline
\end{tabular}

${ }^{*}$ Significant at level $(0.05),{ }^{* *}$ Highly significant at level $(0.01)$.

Table (7): Heritability (on diagonal), phenotypic correlation (above diagonal) and genetic correlation (below diagonal) for average daily gain (ADG) of Japanese quails in second generation of random mating population.

\begin{tabular}{|c|c|c|c|c|c|c|}
\hline Trait & & & $\overline{\mathrm{A}]}$ & & & \\
\hline Ages & $\begin{array}{l}\text { Hatching- } \\
1^{\text {st }} \text { week }\end{array}$ & $\begin{array}{l}1^{\text {st }} \text { week- } \\
2^{\text {nd }} \text { week }\end{array}$ & $\begin{array}{l}2^{\text {nd }} \text { week- } \\
3^{\text {rd }} \text { week }\end{array}$ & $\begin{array}{l}3^{\text {rd }} \text { week- } \\
4^{\text {th }} \text { week }\end{array}$ & $\begin{array}{l}4^{\text {th }} \text { week- } \\
5^{\text {th }} \text { week }\end{array}$ & $\begin{array}{l}5^{\text {th }} \text { week- } \\
6^{\text {th }} \text { week }\end{array}$ \\
\hline Hatching- $1^{\text {st }}$ week & 0.50 & 0.78 & 0.53 & 0.27 & -0.13 & -0.26 \\
\hline $1^{\text {st }}$ week- $2^{\text {nd }}$ week & 0.85 & 0.63 & $0.53^{* *}$ & $0.21^{* *}$ & $-0.12^{* *}$ & $-0.32^{* *}$ \\
\hline $2^{\text {nd }}$ week- $3^{\text {rd }}$ week & 0.73 & $0.97^{* *}$ & 0.15 & $0.13^{* *}$ & $-0.02^{* *}$ & $-0.25^{*}$ \\
\hline $3^{\text {rd }}$ week- $4^{\text {th }}$ week & 0.56 & $0.29^{* *}$ & $-0.11^{* *}$ & 0.85 & $-0.22^{* *}$ & -0.10 \\
\hline $4^{\text {th }}$ week- $5^{\text {th }}$ week & -0.37 & $-0.31^{* *}$ & $-0.31^{* *}$ & $-0.49^{* *}$ & 1.74 & $-0.01^{* *}$ \\
\hline $5^{\text {th }}$ week- $6^{\text {th }}$ week & 0.01 & $-0.23^{* *}$ & $-0.54^{*}$ & -0.02 & $0.18^{* *}$ & 0.87 \\
\hline
\end{tabular}

${ }^{*}$ Significant at level $(0.05),{ }^{* *}$ Highly significant at level (0.01).

Table (8): Heritability (on diagonal), phenotypic correlation (above diagonal) and genetic correlation (below diagonal) for relative growth rates (RGR)of Japanese quails in first generation of random mating population.

\begin{tabular}{|c|c|c|c|c|c|c|}
\hline Trait & \multicolumn{6}{|c|}{ RGR } \\
\hline Ages & $\begin{array}{l}\text { Hatching- } \\
1^{\text {st }} \text { week }\end{array}$ & $\begin{array}{l}1^{\text {st }} \text { week- } \\
2^{\text {nd }} \text { week }\end{array}$ & $\begin{array}{l}2^{\text {nd }} \text { week- } \\
3^{\text {rd }} \text { week }\end{array}$ & $\begin{array}{l}3^{\text {rd }} \text { week- } \\
4^{\text {th }} \text { week }\end{array}$ & $\begin{array}{l}4^{\text {th }} \text { week- } \\
5^{\text {th }} \text { week }\end{array}$ & $\begin{array}{l}5^{\text {th }} \text { week- } \\
6^{\text {th }} \text { week }\end{array}$ \\
\hline Hatching- $1^{\text {st }}$ week & 0.04 & -0.05 & $-0.31^{*}$ & -0.40 & $-0.07^{* *}$ & -0.46 \\
\hline $1^{\text {st }}$ week- $2^{\text {nd }}$ week & -4.37 & 0.13 & 0.06 & $0.05^{* *}$ & $0.04^{* *}$ & $-0.07^{*}$ \\
\hline $2^{\text {nd }}$ week- $3^{\text {rd }}$ week & $3.20^{*}$ & 6.95 & 0.01 & 0.003 & 0.07 & 0.08 \\
\hline $3^{\text {rd }}$ week- $4^{\text {th }}$ week & -1.19 & $1.00^{* *}$ & 2.24 & 1.03 & -0.0002 & $0.37^{*}$ \\
\hline $4^{\text {th }}$ week- $5^{\text {th }}$ week & $0.31^{* *}$ & $-0.18^{* *}$ & 0.30 & -0.24 & 1.41 & 0.11 \\
\hline $5^{\text {th }}$ week- $6^{\text {th }}$ week & 1.08 & $0.30^{*}$ & -0.92 & $0.14^{*}$ & 0.43 & 0.54 \\
\hline
\end{tabular}

* Significant at level (0.05), ** Highly significant at level (0.01). 
Table (9): Heritability (on diagonal), phenotypic correlation (above diagonal) and genetic correlation (below diagonal) for relative growth rates (RGR) of Japanese quails in second generation of random mating population.

\begin{tabular}{|c|c|c|c|c|c|c|}
\hline \multirow{2}{*}{$\begin{array}{l}\text { Trait } \\
\text { Ages }\end{array}$} & \multicolumn{6}{|c|}{ RGR } \\
\hline & $\begin{array}{l}\text { Hatching- } \\
1^{\text {st }} \text { week }\end{array}$ & $\begin{array}{l}1^{\text {st }} \text { week- } \\
2^{\text {nd }} \text { week }\end{array}$ & $\begin{array}{l}2^{\text {nd }} \text { week- } \\
3^{\text {rd }} \text { week }\end{array}$ & $\begin{array}{l}3^{\text {rd }} \text { week- } \\
4^{\text {th }} \text { week }\end{array}$ & $\begin{array}{l}4^{\text {th }} \text { week- } \\
5^{\text {th }} \text { week }\end{array}$ & $\begin{array}{l}5^{\text {th }} \text { week- } \\
6^{\text {th }} \text { week }\end{array}$ \\
\hline Hatching- $1^{\text {st }}$ week & 0.54 & $0.01^{*}$ & -0.40 & -0.34 & -0.07 & 0.39 \\
\hline $1^{\text {st }}$ week- $2^{\text {nd }}$ week & $0.53^{*}$ & 1.42 & 0.04 & $-0.02^{* *}$ & $0.04^{* *}$ & 0.02 \\
\hline $2^{\text {nd }}$ week- $3^{\text {rd }}$ week & -0.46 & 0.02 & 0.21 & 0.07 & 0.15 & 0.12 \\
\hline $3^{\text {rd }}$ week- $4^{\text {th }}$ week & 0.04 & $-0.32^{* *}$ & 0.06 & 0.50 & -0.04 & $0.30^{* *}$ \\
\hline $4^{\text {th }}$ week- $5^{\text {th }}$ week & -0.06 & $0.01^{* *}$ & 0.48 & -0.19 & 1.87 & 0.12 \\
\hline $5^{\text {th }}$ week- $6^{\text {th }}$ week & 0.24 & $-0.07^{*}$ & 0.12 & $-0.07^{* *}$ & 0.40 & 0.61 \\
\hline
\end{tabular}

* Significant at level (0.05), ${ }^{* *}$ Highly significant at level (0.01).

\section{DISCUSSION}

Similar results on body weights at different ages were obtained by Vali et al. (2005) and Shokoohmaud et al. (2007) who found that significant differences among generation for body weights.

Average daily Gain results agreed with Momoh et al. (2014) who recorded increasing in ADG from hatching till 5 weeks then the mean of 5-6 week for Japanese quail ADG decreased. On the other hand, results obtained by Jones and Hughes (1978) who stated that the average daily gain in Japanese quail was at a maximum $4.8 \mathrm{~g} /$ day during the first period from hatch to 3 weeks of age while the gain was lower during the second period from 3 to 6 weeks of age.

To some extent, similar results for relative growth rate were obtained by Magda et al. (2010) who found that the first generation had the highest significant RGR as $88.42 \%$ for period from two to four weeks.

The increase in heritability estimates across generations indicates increasing additive genetic variance across generations (Falconer, 1989). Heritability estimates of body weights agreed with results obtained by El-Fiky (1991), Aggrey and Cheng (1994), Bahie El-Dean (1994) and Magda et al. (2010). Also, similar results obtained by Devi et al. (2010) who found that the estimates of heritability of body weights from 1 to 4 weeks of age revealed the existence of low to high additive genetic variance in Black (0.14 to 0.55) and Brown (0.09 to 0.51$)$ strains respectively.

The magnitude of correlation depends on the stage of improvement of the population as described by Nestor (1971).

Phenotypic and genetic correlations for body weights results agreed with results obtained by El-Fiky (1991), Mousa (1993) and Magda et al., (2010). Contraindicated results were recorded by Sharaf (1992).

This revealed that body weights at different ages in Japanese quails is influence by pleiotropic effect, so selection for higher body weight at early ages of life would bring about concomitant improvement in body weights at later ages as a correlated response to selection. The phenotypic and environmental correlations were also positive among body weights at different ages indicating that by providing good managemental and climate conditions, the body weights can be further improved (Devi et al., 2010).

Contraindicated results for heritability estimates for ADG were obtained by AboulHassan (1997), Aboul-Hassan (2000) and Abdel-Mounsef (2005) who found that medium heritability estimates for sire. 
Similar results of correlations for ADG were recorded by Aboul-Hassan et al., (1999). Medium to high positive genetic correlations obtained for most of the combinations in both Black and Brown Japanese quails indicated that ADGs at different ages were influenced by pleiotropic genes and linkage and selection for high ADGs at early age would automatically improve the ADGs at later ages as a correlated response to selection (Kumari et al., 2013).

Results for heritability estimates for RGR agreed with results obtained by Mona (2008) and Magda et al., (2010).

\section{REFERENCES}

Abdel-Mounsef, N.A. 2005. Non genetic factors affecting some productive traits in Japanese quail. M.Sc. Thesis Fac. Agric. Al-Azhar Univ. Cairo, Egypt.

Aboul-Hassan, M.A. 1997. Selection for growth traits in Japanese quail. 1Early responses. Mansoura J. Agric. Sci. 22: 101-109.

Aboul-Hassan, M.A. 2000. Comparative study of growth traits in two strains of Japanese quail. Fayoum J. Agric . Res.\&Dev. 14:189-197.

Aboul-Hassan, M.A; El-Fiky, F.A. and Attalah, G.E.Y. 1999. Selection for growth traits in Japanese quai. AlAzhar J.2- Correlated response. Agric. Res. 29: 55-70.

Aggrey, S.E and Cheng, K.M. 1994. Animal model analysis of generic (co) variance for growth traits in Japanese quail. Poult. Sci. 73: 1822-1828.

Bahie El-Dean, M. 1994. Selection indices and crossing as a tool for improvement of meat and egg production in Japanese quail. Ph.D. Thesis. Fac. of Agric. Alex. Univ. Egypt.

Baumgartner, J. 1990. Japanese quail as a laboratory animal (in Solvak)
Publishing House of Solvak Academy of Science, Bratislava.

Becker, W.A. 1985. Manual of Quantitative Genetics (4th Ed.) Academic Enterprises, Pullman, Washington, U. S. A.

Broody, S. 1945. Bioenergetics and growth. Reinhold Pub Crop N.Y., U.S.A.

Devi, K.S., Gupta, B. R, Prakash, M.G., Qudratullah, S. and Reddy, A.R. 2010. Genetic studies on growth and production traits in two strains of japanese quails. Tamilnadu J. Veterinary \& Animal Sciences 6(5): 223-230.

El-Fiky, F.A. 1991. Genetic studies on some economic traits in Japanese quail. Ph.D. Thesis, Fac. Agric. AlAzhar Univ., Cairo, Egypt.

Falconer, D.S. 1989. Introduction to Quantitative Genetics. 3rd ed, Longman Group, Essex, England.

Harvey, W.R. and Bearden, G.D. (1962). Table of expected genetic progress in each of two traits. U.S.A; ARS-20-12.

Jones, J.E. and Hughes, B.L. 1978. Comparison of growth rate body weight and feed conversion between Coturnix DI quail and Bob white quail. Poult. Sci. 57: 1471-1472.

Kumari, B.P., Gupta, B.R., Prakash, M.G., Reddy, A.R. and Reddy, K.S. 2013. Genetic studies on growth rates in japanese quails (coturnix coturnix japonica) Tamilnadu J. Veterinary \& Animal Sciences 9 (2) 122 - 129, March - April 2013.

Magda, I. Abo Samaha, Sharaf, M.M. and Hemeda, Sh. A. 2010. phenotypic and genetic estimates of some productive and reproductive traits in japanese quails. Egypt. Poult. Sci. 30(3): 875892.

Momoh, O.M., Gambo, D. and Dim, N.I. 2014. Genetic parameters of growth, body, and egg traits in Japanese quails 
(Cotournix cotournix japonica) reared in southern guinea savannah of Nigeria. Journal of Applied Biosciences 79: 6947 - 6954.

Mona, E. Younis 2008. Selection against obesity in Japanese quails. M. V. Sc. Animal breeding and production. Fac. of Vet. Med. Alex. Univ.

Mousa, K.R.M. 1993. The influence of different nutritional conditions on some genetic parameters in Japanese quail. M.Sc. Thesis, Fac. Agric. AlAzhar, Univ. Cairo, Egypt.

Nestor, K.E. 1971. Genetics of growth and reproduction in the turkey. 3. Further selection for increased egg production. Poult. Sci. 50: 16721682.

Panda, B., Singh, R.P. 1990. Development in processing quails. World Poult. Sci. Journal, 46: 219-234.
SAS (2002): SAS/STAT users guide. SAS Institute INC, Cary, NC 27513, USA.

Sharaf, M.M. 1992. Genetic and non genetic estimates of some reproductive and productive traits in Japanese quail. Egypt. Poult. Sci. 12: 211-231.

Shokoohmaud , M., Kashan, N.E.J. and Maybody, M.A.E. 2007. Estimation of heritability and genetic correlations of body weight in different age for three strains of Japanese quail. International Journal of Agriculture and Biology. 9(6): 945 - 947.

Vali, N., Edriss, M.A. and Rahmani, H.R. 2005. Genetic parameters of body and some carcass traits in two quail strains. Int. Poult. Sci. 4(5): 296-300. 